\title{
CREATIVE CONNECTIVITY PROJECT - A NETWORK BASED APPROACH TO UNDERSTAND CORRELATIONS BETWEEN INTERDISCIPLINARY GROUP DYNAMICS AND CREATIVE PERFORMANCE
}

\author{
Vanna Savina TORRISI ${ }^{1}$, Sabato MANFREDI ${ }^{2}$, lacopo IACOPINI ${ }^{3,4}$ and Vito LATORA ${ }^{3,4,5}$ \\ ${ }^{1}$ School of Design, Royal College of Art \\ ${ }^{2}$ Department of Electrical Engineering and Information Technology, University of Naples \\ Federico II \\ ${ }^{3}$ School of Mathematical Sciences, Queen Mary University London, London E1 4NS, UK \\ ${ }^{4}$ The Alan Turing Institute, The British Library, London NW1 2DB, UK \\ ${ }^{5}$ Dipartimento di Fisica, Università di Catania and INFN, I-95123 Catania, IT
}

\begin{abstract}
Creativity, technology and innovation are fundamental driving forces that often trigger behavioural and cultural changes in our societies. Several studies on creative collaborations emphasise the interconnection between the creation of innovation and interdisciplinarity. Studying the interdisciplinary processes through which innovation is generated is thus of fundamental importance. The Creative Connectivity project investigates the links between group dynamics and interdisciplinary creative processes in the attempt to identify the emergence of meaningful behavioural patterns. Taking the Innovation Design Engineering (IDE) programme at the Royal College of Art as a case study, the research addresses the need to develop a more rigorous understanding of the creation of innovation fostered by diversity, a risk-taking culture and acceptance of failure. A novel approach combining data science and network theory has been developed to monitor, quantify and analyse specific dynamics of interdisciplinary groups of students at the IDE programme. The focus was to investigate the network contribution to the performance of a team in an environment that is naturally interdisciplinary by using data respectively from a bespoke developed web application and a conversation activity monitoring system. Initial findings have shown the potential of this approach to unveiling the hidden mechanisms behind creativity and the production of innovation through interdisciplinary approaches. The results of this research could be of interest for universities, research centres, start-ups incubators, and policymakers who want to foster creativity and trigger innovative processes.
\end{abstract}

Keywords: Interdisciplinary process, innovation, network theory, group dynamics, connectivity

\section{INTRODUCTION}

Research studies explore several aspects of the strong correlation between innovation and interdisciplinarity. The latter arises to embrace the increasing complexity of the present and the future that innovation aims to address to create value for societies [1]. Interdisciplinarity is a way of thinking, a modus operandi, and, as Newell asserts, a distinctive process defined by the complex nature of its objects of study that "[...] involves connecting part to part, part to whole, and whole to part" [2]. Interdisciplinary processes generate systems of not obvious correlations and interactions fostering the creation of breakthrough innovations. Unlike twenty years ago, the invention of newness does no longer focus on one single ground-breaking idea [3]. Instead, it is the result of an evolutive process of constant contamination deployed by intercorrelated ideas generated by connected minds; a networked system of complex rather than precise connections that embrace the unconventional while fostering deviations from linear creative processes [4]. The intricate correlations between three main aspects seem to define the creation of impactful innovation: the unfolding of the design process, the group dynamics and their impact on the quality and originality of the final outputs. This research attempts to explore the above-mentioned interconnections and identify the emergence of patterns of behaviours 
through the lens of the Innovation Design Engineering programme at the Royal College of Art (RCA). The programme's philosophy is based on a learning culture fostered by openness to the unfamiliar, ability to engage with each other's diversity while enabling miscommunication and tension to emerge as powerful driving forces. This approach to radical innovation emphasises the emergence of independent and critical thinking fostering originality through interdisciplinary collaborations based on a risk-taking culture and acceptance of failure. [5]

\section{A REVIEW OF EVIDENCE IN THE FIELD}

Connectivity, exposure to failure and hybridisation are factors inherently embedded in innovative thinking. Steven Johnson points out "If you want to create a space for innovation you won't get far by cloistering yourself away from the world and waiting for inspiration. Chance favours the connected mind." [6].

Creativity aiming at the development of real innovation is a process of hybridisation that involves decontextualisation and recontextualisation of knowledge resulting in valuable novel insights. Ronald Burt, who describes creativity as a process involving knowledge, incubation, networking, exchange and transformation, asserts, "People who live in the intersection of social worlds are at higher risk of having good ideas" [7]. Hybridisation implies the merging of diverse identities, the presence of a border to be overlapped and uncertainty. Beck stresses that dealing with 'chronic uncertainty' culminates in the perception of risk as an avoidable and a desirable factor [8]. This approach fosters openness to evolution, and therefore to innovation while exponentially increasing the chance of failure [1]. Fleming in his 'Perfecting Cross Pollination', research on 17,000 patents, affirms that the bigger is the distance between the team members' fields the lower is the quality of their innovations. However, as illustrated in the exhibit "Going for Breakthrough", interdisciplinary collaborations generate breakthroughs of higher value than the novelty delivered by teams of people from similar backgrounds. Fleming's observation suggests that key innovation strategies could be implemented "[...] to manipulate the makeup of an innovation team to achieve a desired balance between risk and reward". Bringing together individuals with deep, rather than broad expertise in very diverse fields seems to expose the team to a lower risk of failure [9]. However, a number of case studies indicate that a group with shallow expertise, but inquisitive attitude could produce a broad array of highly original yet unrefined ideas at the early stages of the creative process [5]. As observed in the Re-mix research project, the specialist knowledge of the Kyushu University participants combined with the diverse and broad approach to creativity of the RCA participants had a major impact on the final phase of the project and enabled to deliver refined outputs in a considerably short period [1]. These insights reinforce the authors' belief that identifying emergent patterns of behaviours in interdisciplinary collaborations could enable to orchestrate the group dynamics and the design process without spoiling the creative momentum.

\section{TOOLS}

A series of research projects run at the RCA in collaboration with international institutions in the last seven years [1][5] has produced the following observations and shed light on interdisciplinary dynamics: (a) Horizontal team structure fosters prompt reconfiguration of team dynamics and spontaneous emergency of leadership between team members; (b) Correlation between the level of agreement amongst team members and the individual's level of satisfaction with the latter not explicitly related to the quality of the final output; (c) Excess verbal communication and lack of actions leading to conflictual team dynamics point to a deep crisis within teams; (d) Intense sketching and model making activities replace excess verbal communication as a reaction to the experienced crisis. (e) A higher level of agreement and more cohesive collaboration among team members are encountered when approaching the final deadline.

In the attempt of developing a novel approach that aims to monitor and analyse interdisciplinary group dynamics, a set of tools was created. The system that combines the collection of qualitative and quantitative data included:

- a bespoke web application to issue daily questionnaire

- a conversation activity monitoring programme to retrieve quantitative data regarding the frequency of messages exchange within each group via a Slack group account. 
The daily questionnaires aimed at fostering self-reflection on the design process and improve group dynamics by enabling each student to evaluate their performance and those of the other members of their team. A five-point Likert scale - 1: low level, 5: high level - complemented by open text was used to rate each team members according to the following criteria:

- Group productivity

- Interaction among other group members

- Level of agreement with other group members

- $\quad$ Satisfaction towards the group's design process - self-evaluation only

In addition to the above, five-point Likert scales, where used to rate the following features to monitor the emergence of patterns of personality traits and related behaviours over the course of the project:

- $\quad$ Leader / Follower - 1 respectively referred to Leader and 5 to Follower

- Thinker / Maker - 1 respectively referred to Thinker and 5 to Maker

The evaluating system did not imply any positive or negative connotation associated with any of the personality traits mentioned above. The conversation monitoring system was intended to explore the correlation between the intensity of the exchange of verbal communication and the level of agreement/disagreement as observed in previous research projects focusing on creative interdisciplinary collaborations.

\section{PROJECT}

Creative Connectivity is a research project that attempts to unveil the inherent dynamics of interdisciplinary collaborations by intersecting fields of knowledge like design thinking and mathematical theories, that are practised at great distances from one another.

Forty-seven 1st year students of the Innovation Design Engineering dual masters programme at the Royal College and Imperial College London were clustered in groups of four to undertake the Gizmo course that combines physical computing and mechatronics to create playful, devious or absurd machines as shown in Fig.1. The group forming process was based on the dual criteria of clustering together students from diverse disciplinary backgrounds and who have not worked together in previous modules. The students' multidisciplinary background, the short length of the project and the challenging brief delivered a fast-paced and dynamic learning experience involving experimentation, intense prototyping, a risk-taking attitude and hectic collaborative interactions.

A panel of tutors assessed the final outputs and teams' performance based on the following criteria: Functionality of machine, Inventiveness and Creativity, Engineering Design \& Refinement, Aesthetics of machine, Code layout, Team's verbal and visual presentation skills.
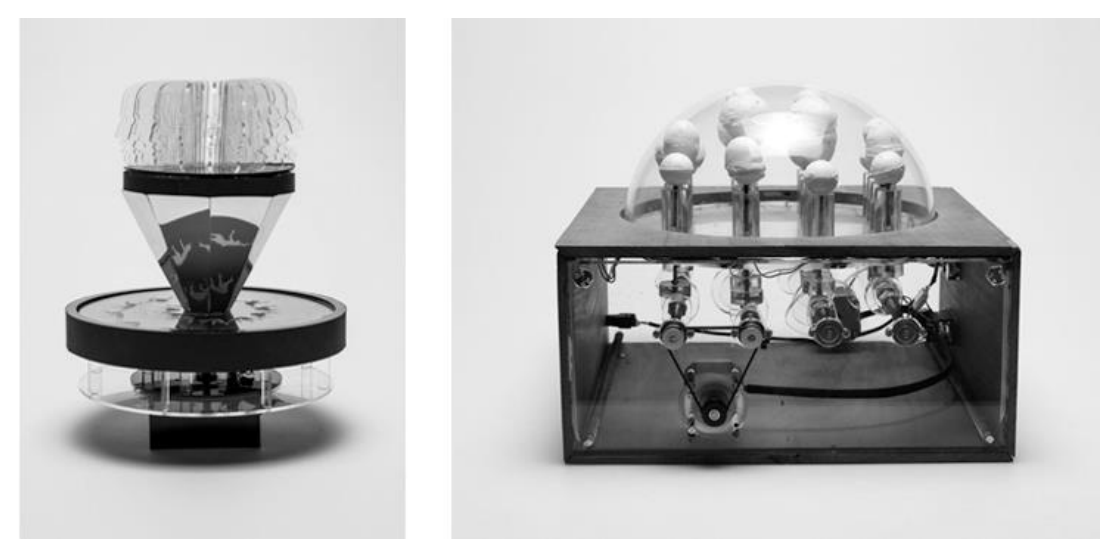

Figure 1. a). The 'Curio' machine designed by B. Ching, F. Weil, G. Whittembury and K. Zhang. (b) The 'Loligo' machine designed by S. Chestler, J. Fraser, Y. Hung and S. Suzuki

Due to the following factors, some students underused the set of tools described in section 3:

- Very dense module schedule involving the acquisition of new knowledge and skills in a short period perceived as not compatible with daily completion of questionnaires and online exchange of information

- Students reluctant to be monitored for research purposes 
- Mutual rating between all team's member recognised as a source of tension rather than a selfreflective tool enabling the improvement of group dynamics, especially if used during the course of the design process instead of at the end of it.

The conversation activity monitoring programme did not succeed in delivering valuable insights on the online messages exchanged between team members. The fast-paced nature of the module fostered face-to-face conversations, recognised instead as more effective than when mediated by an interface. However, the gathered questionnaire data still enabled to reveal valuable insights regarding the dynamics of interdisciplinary collaboration.

\section{ANALYSIS OF DATA}

In what follows we will present an analysis aimed to explore correlations between creative performance and group dynamics.
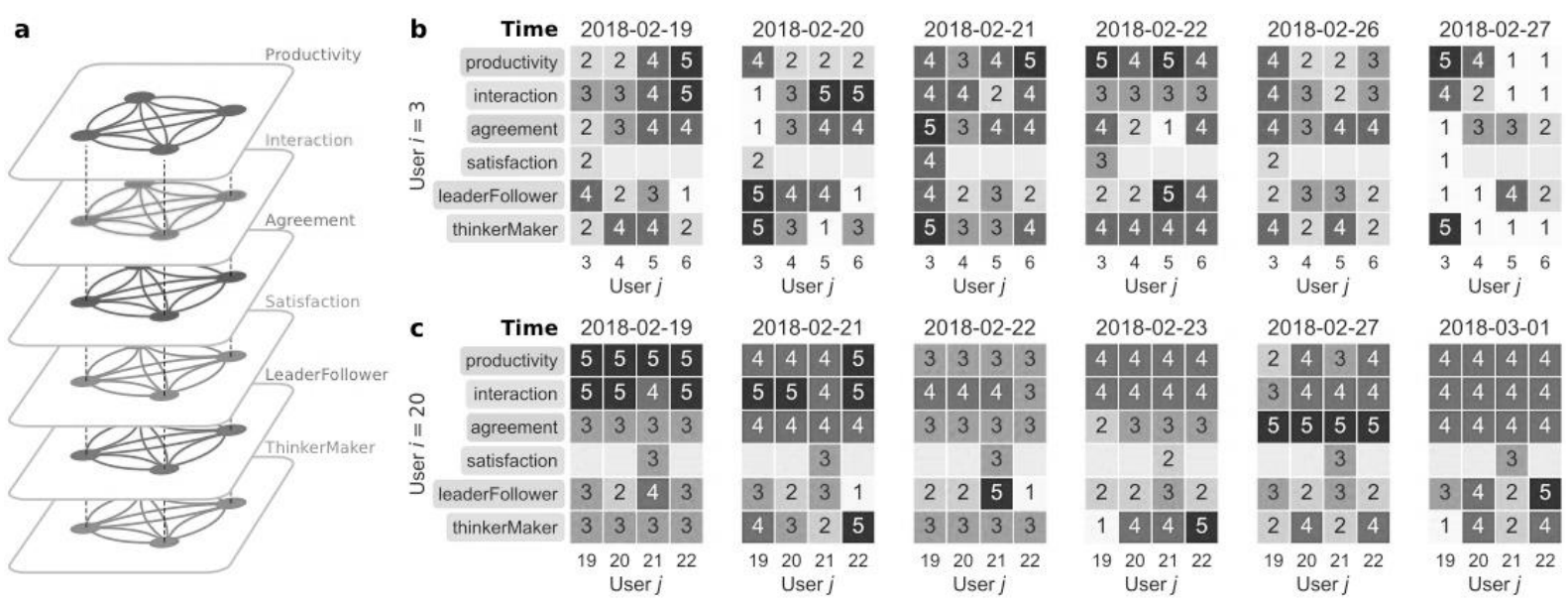

Figure 2. a) Multiplex network representation of group interactions, where each layer is associated to a different feature. The time-resolved nature of the user submissions allows us to create temporal snapshots of the multiplex networks. (b-c) Two examples for users 20 and 3 are shown

Interactions among the individuals within each group can be represented as time-varying multiplex networks, i.e. networks where the links can be of different types (the so-called layers of a multiplex network) and can change in time $[10,11,12]$. In the case under study the $N$ members of a group are described as the nodes of the network and are linked to each other across $M$ different layers, one for each feature (see Figure 2a). In particular, the data allowed to assign a weight (numerical value in the range $[1,5]$ ) to each directed link between two users of the same group, according to the corresponding value declared in the questionnaires. This has been done for each group, for each of the five features discussed in the previous section (i.e. Productivity, Interaction, Agreement, Leader/Follower, Thinker/Maker), and for each time. Given a group $g$ and two users in the group $i, j \in g$, we denote with $w_{i j}^{[\alpha]}(t)$ the value that user $i$ assigned to the user $j$ at time $t$ with respect to the feature $\alpha$. Examples of the values assigned by two specific users ( $i=3$ and $i=20)$ are shown in Figure $2 \mathrm{~b}$ )-c).

In order to compare the student self-evaluation with the one assigned by others members of a group, we compare the self-score of a feature $\alpha$ for a user $i$ given by $\left\langle w_{i i}^{[\alpha]}(t)>_{\mathrm{t}}\right.$ with the average score that user $i$ received by the other group members, given by $\frac{1}{N-1} \sum_{\substack{j \in g \\ j \neq i}}<w_{j i}^{[\alpha]}(t)>_{\mathrm{t}}$. Notice that both quantities are also averaged over time, with $\langle\cdot\rangle_{t}$ denoting the temporal average. Results for the different features are shown in Figure 3. For some features, such as Productivity and Leader/Follower features, the individual perceptions seem to be in accordance with the ones assigned by the rest of the group. The same cannot be said for the Interaction feature. The consistency of the perception of leadership within a group might be correlated to the horizontal team structure. It seems to foster the spontaneous emergence of leadership that is dictated by a mix of personal skills and personality traits. The impulsive nature of this phenomenon seems to encourage the effective sharing of responsibilities and leadership according to specific phases of the design process. 

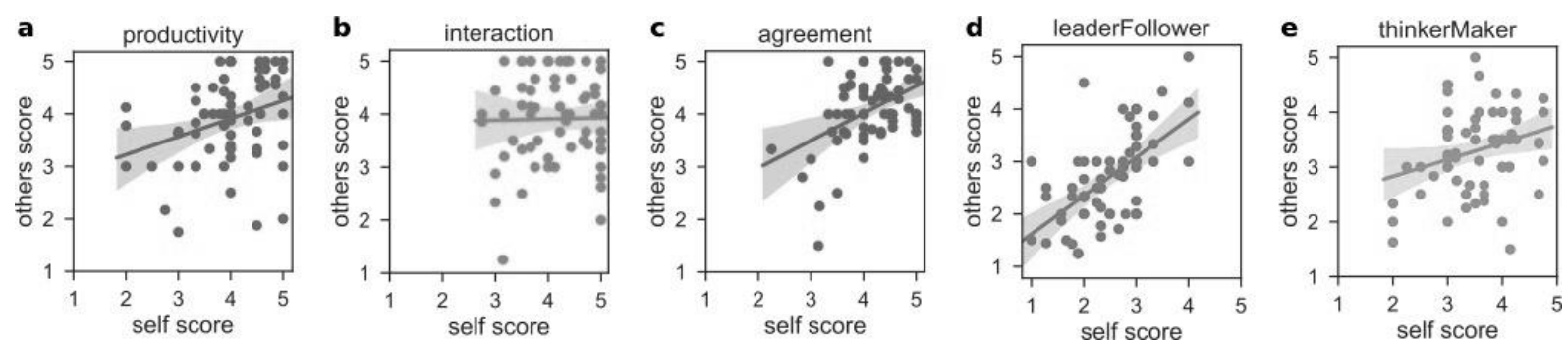

Figure 3. Scatter plots of self-perception versus perception from others for each of the five considered features

We explore now the relationship between the features mutually assigned by group members during the development phase of the projects and the final score that the project received at the end. Figure 4 shows the boxplots for three features, where both the self-score (red) and the score received from team members (blue) are grouped with respect to the final mark of the projects, as given by external examiners. In the case of the two features of Productivity and Interaction (panels a-b) we notice an inverse correlation between their values and the final score assigned to the group by the examiners. This is true both for the self (red) and the mutual (blue) values. More specifically, extreme marks can provide interesting insights. For example, projects of students who declared high values of productivity for themselves and for the other group members received the lowest marks by the external examiners. This behaviour seems to confirm what encountered in previous projects where the individual's level of satisfaction was aligned with the perceived level of agreement within the group but had no coherent link with the quality of the final output and therefore with the real productivity of the team.
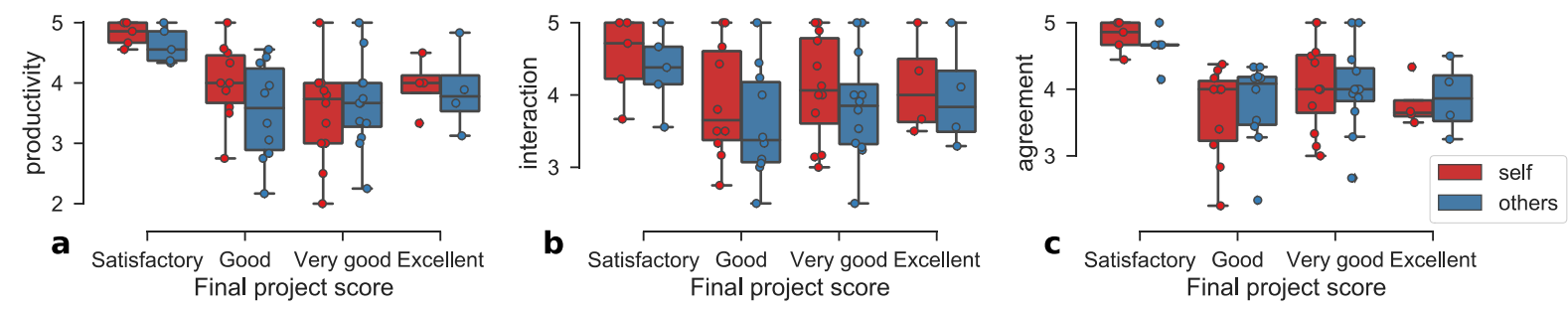

Figure 4. Comparison of group self- and others- perception with final project score

Finally, we look at the temporal evolution of the features of two specific users. Figure 5 shows, for a user $i$, the temporal behaviour of the self-score $w_{i i}^{[\alpha]}(t)$ associated to a given feature $\alpha$ (dashed lines), together with the average score that the same user assigned to the other members of the group for the same feature, that reads $\frac{1}{N-1} \sum_{\substack{j \in g \\ i \neq j}} w_{i j}^{[\alpha]}(t)$ (continuous lines). We can first observe a general trend of the two users in assigning lowest scores to the other than to themselves, as it emerges from the comparison of the dashed and the continuous lines. Secondly, the fluctuation associated with the average score given by students to their peers seems to increase after the first two days of the project (shaded region denoting the standard deviation from the mean values). This might represent the phenomena of increasing of group collaboration during the intermediate phase of the project and mirror what observed in previous projects as stated in section 3 .

Teams members seem to achieve an increased level of agreement regarding design choices and projects objectives through a renewed collaborative attitude when approaching the final deadline.
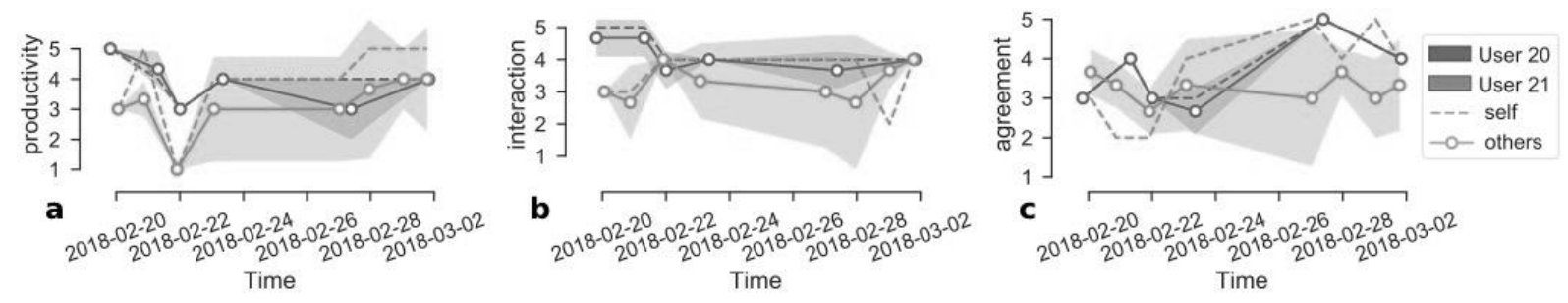

Figure 5. Temporal evolution of self-assigned and received scores for three different features. The cases of two students are shown 


\section{FINDINGS}

The Creative Connectivity project has demonstrated the strong potential of the novel approach that combines data science and network theory to gain new insights regarding interdisciplinary creative collaborations and the emergence of significant patterns of behaviours in design processes. The analysis of the data collected through a bespoke web application revealed valuable insights and identified the emergence of the following behaviours:

- The consistency of the perception of leadership within groups appears to be correlated to the horizontal team structure. Several studies underline that the non-hierarchical structure of the team seems to foster the spontaneous emergence of leadership as well as the effective sharing of responsibilities according to specific phases of the design process.

- Consistent perception of the level of productivity within teams seems to have an inverse correlation with the quality of the final output as assessed by external examiners according to the criteria listed in section 4. This behaviour might suggest a lack of objectivity towards the effectiveness of the team members' collaboration.

- More cohesive collaboration is experienced from the intermediate phase of the project. An increasing level of agreement on the design choices is often encountered in interdisciplinary collaborations when approaching final deadlines and, as observed in previous projects, teams tend to replace excess verbal communication with intense model making.

The students' feedback on the tools described in section 3 led the authors to believe that minimising the peer to peer approach based on the mutual rating of team members' performance in favour of a more self-reflective approach could yield a broader consensus, while still delivering valuable data. Further research is required in the development of the tools, with particular emphasis on the effectiveness of the approach enabling the rigorous collection of data without interfering with the natural course of the design process. The above findings support the authors' belief that identifying and analysing emerging patterns of behaviours in interdisciplinary collaborations could be fundamental to develop a new understanding of team dynamics and its strategic manipulation. These preliminary results could be of interest for universities and research laboratories interested in pursuing the creation of impactful innovation.

\section{REFERENCES}

[1] Torrisi V.S., Inamura T. and Hirai Y. Re- mix Project - Hybridisation Leading to Disruptive Innovation. In International Conference on Engineering and Product Design, EPDE'18, London.

[2] Newell W.H. The State of the Field: Interdisciplinary Theory. Issues in Interdisciplinary Studies, 2013, 31, pp.22-43

[3] Jones R. From Knowledge Production to Wisdom Creation; Transformation in the Post disciplinary Era. Available at http://www.icelanddesign.is/NEWSANDEVENTS/Readarticle/2640 [Accessed 8March 2018]

[4] Iacopini I., Milojević S. and Latora V. Network Dynamics of Innovation Processes, Phys. Rev. Lett. 120, 048301 (2018)

[5] Torrisi V.S. and Hall A. Missing Miscommunication in Interdisciplinary Design Practice. In International Conference on Engineering and Product Design, EPDE'13, Dublin, 2013

[6] Johns S. Where Good Ideas Come From - A Natural History of Innovation, 2010 (Penguin Group).

[7] Burt R. Structural Holes and Good Ideas, American Journal of Sociology110, September 2014

[8] Storni C., Lee Y., Schepers S. and Schofeelen J. Participation Is Risky: Approaches to Joint Creative Processes, 2014 (Valiz/Antennae Series).

[9] Fleming L. Perfecting Cross- Pollination, Harvard Business Review, September 2004

[10] Nicosia V., Tang J., Mascolo C., Musolesi M., Russo G. and Latora V. in Temporal Networks. Graph Metrics for Temporal Networks, Edited by Holme P. and Saramaki J. Springer, page 1540, 2013

[11] Latora V., Nicosia V. and Russo G. Complex Networks: Principles, Methods and Application, Cambridge University Press, 2017.

[12] Manfredi S. Multilayer Control of Networked Cyber-Physical Systems. Application to Networked Monitoring, Autonomous and Robot Systems in Advances in Industrial Control, Springer, 2017 